\title{
Pressure-induced Structural and Hydration Changes of Proteins in Aqueous Solutions
}

\author{
Min Zhang and Yuqing Wu $\mathbf{U}^{\dagger}$ \\ State Key Laboratory for Supramolecular Structure and Materials, Jilin University, No. 2699 Qianjin Street, \\ Changchun 130012, P. R. China
}

\begin{abstract}
The effects of elevated hydrostatic pressure on four representative proteins, lysozyme, human serum albumin, ubiquitin and RNase A, were investigated by using Fourier transform infrared (FTIR) spectroscopy, by principal component analysis (PCA) and by moving-window two-dimensional (MW2D) correlation analysis. In addition, we revealed the pressure-induced changes of secondary structure elements using curve fitting. With pressure increase, the amide I band shifted to lower wavenumbers, with a transition at $200 \mathrm{MPa}$, which was indicative of hydration enhancement. Moreover, the pressure-induced behavior of pure water was studied, similar transition pressure was observed with protein in aqueous solution, suggesting that structure change of water around $200 \mathrm{MPa}$ caused a hydration enhancement of protein. Under pressure higher than $200 \mathrm{MPa}$, the structural changes of the four proteins were obviously different except for the common features shifting to lower wavenumbers with pressure, basically due to the distinct structural differences among them.
\end{abstract}

(Received August 17, 2011; Accepted September 2, 2011; Published November 10, 2011)

\section{Introduction}

The mechanisms of protein folding and unfolding have been investigated in intensive experimental and theoretical studies for several decades. Furthermore, a detailed understanding of the structure in the unfolded state of proteins has been a major research topic in protein chemistry and biophysics in recent years. Pressure as well as temperature induced effects in proteins are closely connected with the presence of water. Changes in the interaction with water (hydration) and the imperfect packing effects in the interior (void volume and cavities) contribute greatly to the structural and dynamic variations of proteins in aqueous solutions. ${ }^{1-3}$

Pressure is able to change the shape of a protein, extremely high pressure can lead to unfolding of the protein, but lower pressures cause only elastic distortion of the secondary and tertiary structures. ${ }^{4,5}$ In order to clearly explain the relationship between structure changes of water and protein, we chose four typical proteins, lysozyme, human serum albumin (HSA), ubiquitin and RNase A as samples, these have different secondary structures and space conformations. Lysozyme mainly contains $\alpha$-helices and subsidiary $\beta$-sheets. ${ }^{6,7}$ HSA mainly includes $\alpha$-helices and minor random coils. ${ }^{8}$ Ubiquitin mainly contains $\beta$-sheets and subsidiary $\alpha$-helices. ${ }^{9} \quad$ RNase A with a $\beta$-sheet main structure, contains $\alpha$-helices and turns. ${ }^{10}$ We investigated pressure-induced structure changes of four proteins in aqueous solution from 0.1 to $740 \mathrm{MPa}$ by using infrared spectroscopy. Both the hydration and minor structural changes of proteins have been revealed clearly by principal component analysis (PCA) and moving-window two-dimensional (MW2D) correlation analysis.

$\dagger$ To whom correspondence should be addressed.

E-mail: yqwu@jlu.edu.cn

\section{Experimental}

\section{Reagents and chemicals}

Lysozyme, HSA, ubiquitin, RNase A and deuterated Tris-DCl (Tris(hydroxymethyl)aminomethane) were purchased from Sigma Chemical Co.; these materials were used without further purification. Deuterium oxide $\left(\mathrm{D}_{2} \mathrm{O}\right)$ was purchased from Cambridge Isotope Laboratory, Inc. The concentrations of proteins were $50 \mathrm{mg} / \mathrm{ml}$ in $50 \mathrm{mM}$ Tris/ $\mathrm{D}_{2} \mathrm{O}$, followed by a $\mathrm{pD}$ 7.8. Before the pressure scan, the samples were heated up to $45^{\circ} \mathrm{C}$ for $30 \mathrm{~min}$ and then cooled down to enable the complete H/D exchange of all solvent-accessible protons. ${ }^{11}$

\section{Apparatus and procedure}

High pressure was generated in a diamond anvil cell (Diacell Lever DAC-Mini A65000), where the pressure was built up by means of a screw mechanism. ${ }^{12}$ The $\mathrm{BaSO}_{4}$ was the internal pressure calibrant, and the IR absorption band is $983 \mathrm{~cm}^{-1} .^{13}$ Infrared spectra were measured at room temperature on a Bruker Vertex 80v Fourier transform spectrometer equipped with a liquid nitrogen cooled mercury cadmium telluride detector. For each spectrum, 512 interferograms were co-added with a spectral resolution of $2 \mathrm{~cm}^{-1}$. The obtained spectra were baseline corrected in the region of the amide I band. ${ }^{14,15}$ Curve fitting with Gaussian functions was performed using Origin software. PCA was performed with the treated spectra after mean-centering using the Unscrambler 5.0 program. MW2D correlation plots were computed using 2D Shige software. ${ }^{16}$

\section{Results and Discussion}

Pressure-induced structural changes of four proteins

The structural changes of lysozyme are displayed in Fig. 1A. 


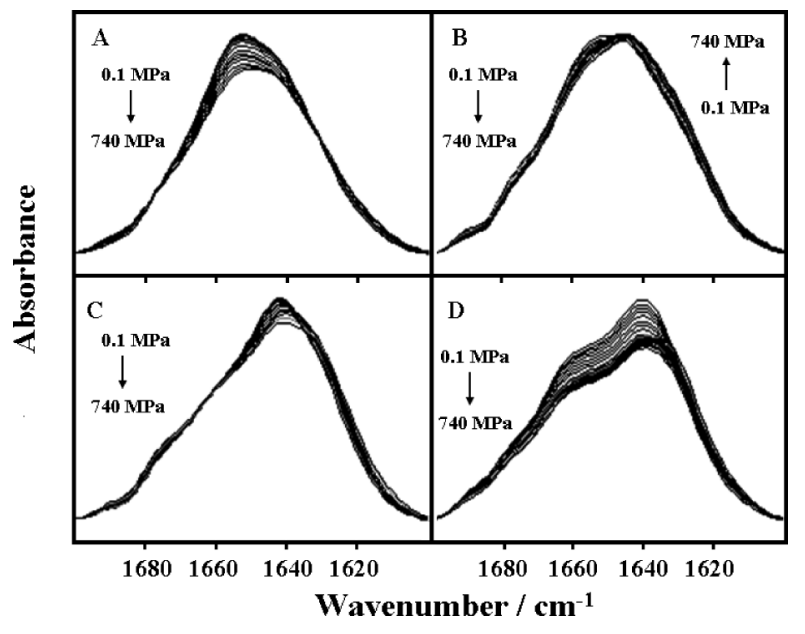

Fig. 1 Pressure-induced IR spectra of (A) lysozyme, (B) HSA, (C) ubiquitin and (D) RNase A. Arrows represent the direction of change with increasing pressure.

Table 1 Fraction of secondary structure elements of four proteins in the initial and final state, respectively

\begin{tabular}{lccccc}
\hline & & \multicolumn{3}{c}{ Population of secondary structure elements } \\
\cline { 3 - 6 } Protein & P/MPa & $\alpha$-Helix, \% & $\begin{array}{c}\beta \text {-Sheet, } \\
\%\end{array}$ & $\begin{array}{c}\beta \text {-Turn, } \\
\%\end{array}$ & $\begin{array}{c}\text { Random } \\
\text { coil, \% }\end{array}$ \\
\hline Lysozyme & 0.1 & 47.7 & 18.5 & 15.7 & 18.2 \\
& 740 & 18.4 & 19.1 & 35.7 & 26.8 \\
HSA & 0.1 & $52.1^{\mathrm{a}}, 29.3^{\mathrm{b}}$ & & 8.1 & 6.8 \\
& 740 & $45.5^{\mathrm{a}}, 41.0^{\mathrm{b}}$ & & 5.6 & 5.5 \\
Ubiquitin & 0.1 & 21.4 & 45.9 & 21.5 & 11.1 \\
& 740 & 12.1 & 56.0 & 25.6 & 6.3 \\
RNase A & 0.1 & 20.0 & 46.1 & 28.6 & 5.3 \\
& 740 & 11.9 & 41.1 & 35.7 & 11.2 \\
\hline
\end{tabular}

a. The solvent-inaccessible $\alpha$-helix.

b. The solvent-exposed $\alpha$-helix.

Under the initial pressure, the amide I band is dominated by 1656 and $1639 \mathrm{~cm}^{-1}$, which are assigned to $\alpha$-helix and the $\beta$-sheet, respectively. ${ }^{6,7}$ During the pressure increase, the band at $1656 \mathrm{~cm}^{-1}$ shifts to around $1643 \mathrm{~cm}^{-1}$. The spectrum of the native HSA has a main peak at $1656 \mathrm{~cm}^{-1}$, with shoulders at 1636 and $1673 \mathrm{~cm}^{-1}$, the positions consistent with the crystallographic structure of HSA. ${ }^{13,17,18}$ As the pressure increases, the maximum gradually shifts to the lower wavenumbers. The band at $1639 \mathrm{~cm}^{-1}$ decreased with pressure in Fig. 1C, and shifted to lower wavenumbers. Under initial pressure, the amide I band of RNase A are dominated by the $\beta$-sheet at $1641 \mathrm{~cm}^{-1}$ and a turn at $1663 \mathrm{~cm}^{-1}$. With pressure increase, weakening of the band at $1663 \mathrm{~cm}^{-1}$ and a shifting from $1641 \mathrm{~cm}^{-1}$ to lower wavenumbers occur; such band features can be observed more clearly in the second derivative spectra (Fig. S1, Supporting Information).

The curve fitting (Fig. S2, Supporting Information) was performed and is shown in Table 1. It clearly displays that the $\alpha$-helix content in lysozyme decreases $29.3 \%$, the turn and random coil increase 20.0 and $8.7 \%$, respectively, but the $\beta$-sheet decreases slightly. In the HSA, the solvent inaccessible $\alpha$-helix decreases $6.7 \%$ accompanied with an $11.8 \%$ increase of the solvent exposed $\alpha$-helix, but the turn and random coil change slightly. The $\alpha$-helix and random coil of ubiquitin

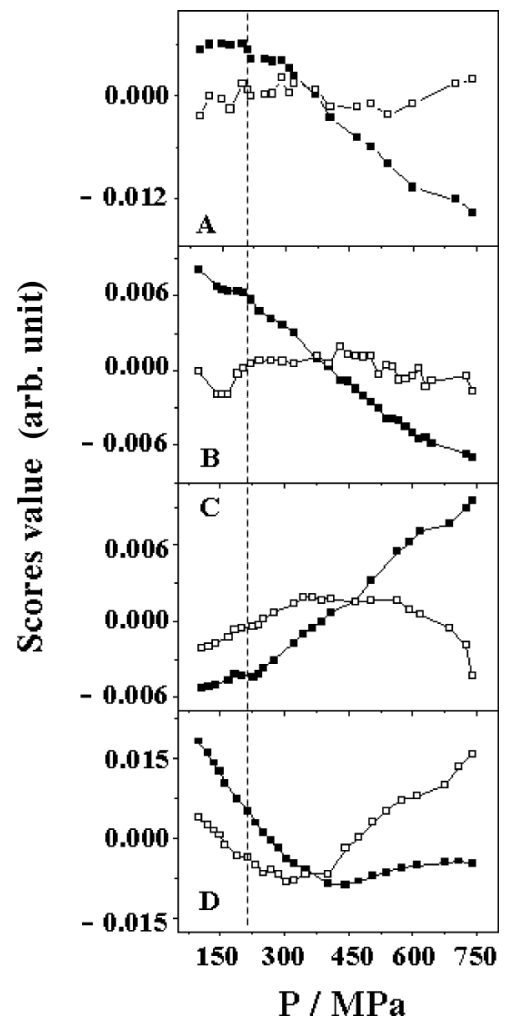

Fig. 2 Score plots of the first two principal components $(\mathbf{\square}, \mathrm{PC} 1 ; \square$, PC2) for (A) lysozyme, (B) HSA, (C) ubiquitin and (D) RNase A.

decrease 9.3 and $4.8 \%$, respectively, while the $\beta$-sheet and turn increase $14.4 \%$ in total. The main structure is $\beta$-sheet in RNase A, it decreases $4.9 \%$, while the turn and random coil increase $13.0 \%$ in total.

The random coil increases with pressure in the lysozyme and RNase A, which is opposite to the HSA and ubiquitin cases, implying that a pressure induced structural change in proteins does not always transfer from a regular structure to a random one. In addition, the regular secondary structures in proteins like HSA and ubiquitin would rearrange from the original one under pressure, leading to a secondary structure, which is more stable than the native one under pressure.

Pressure induced dynamics of four proteins as revealed by PCA

PCA can evaluate some information in the directions of both the spectral variables (loading plot) and the perturbation (score plot) individually. ${ }^{19}$ Figure 2 shows score plots of the first two important PC factors, which account for nearly $100 \%$ of the total variance. The curve of PC1 in Fig. $2 \mathrm{~A}$ is a plurality state process, with several transitions at about 200, 400 and $580 \mathrm{MPa}$. Further considering the scores of PC2, despite the fact that PC2 accounts for only $2 \%$ of the total variance, one can observe two significant transition pressures at 200 and $580 \mathrm{MPa}$, can be observed by $\mathrm{PC} 2$. The second transition $(P=580 \mathrm{MPa})$ in PC2 correlates well with that observed from the $\mathrm{PC} 1$, in agreement with the result of the band analysis (Fig. S3A, Supporting Information).

The two PCs of HSA is not a pure two-state process, several fluctuations appeared at around 200, 400, 500 and $600 \mathrm{MPa}$. Although PC2 accounts for only $5 \%$, one significant change appears at $200 \mathrm{MPa}$. The PCA results of ubiquitin in Fig. 2C, similarly, for the curve of PC1-scores $(89 \%)$ not a two-state process is evident. The PC2 $(11 \%)$ value for ubiquitin is 


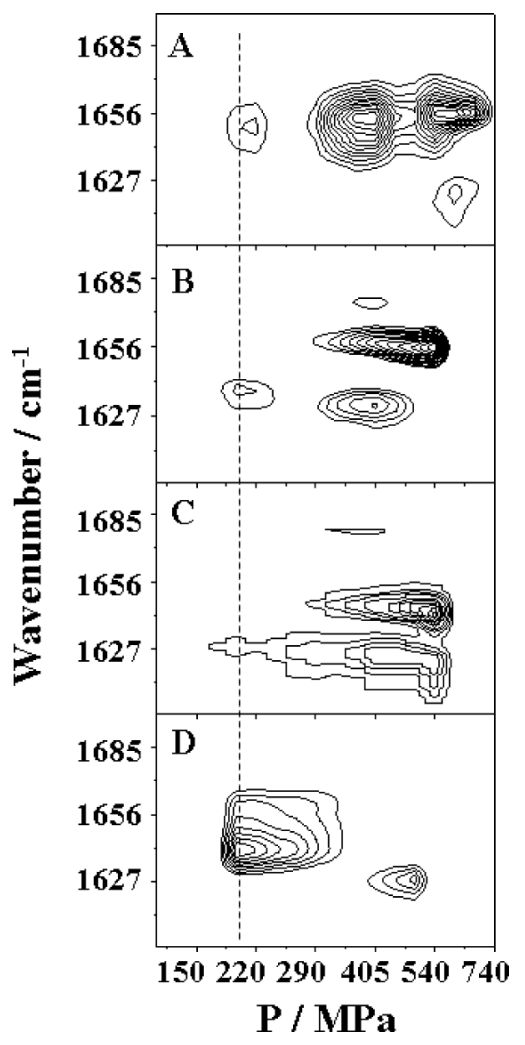

Fig. 3 MW2D correlation spectra of (A) lysozyme, (B) HSA, (C) ubiquitin and (D) RNase A.

relatively higher than the value for other proteins, which can be attributed to the changes at around 200 and $680 \mathrm{MPa}$, respectively. Compared with lysozyme, HSA and ubiquitin, the PC1 curve $(85 \%)$ of RNase A is more approximated by a two-state process, with a midpoint at $P_{\mathrm{m}}=400 \mathrm{MPa}$. A slight fluctuation at around $200 \mathrm{MPa}$ in the $\mathrm{PC} 1$ curve, combined with the $15 \%$ variance explaining PC2, illustrate the presence of two transitions at around 200 and $400 \mathrm{MPa}$.

Moving-window $2 D$ correlation analysis reveals the hydration revealing of four proteins

We applied MW2D correlation analysis to these spectra. ${ }^{20}$ As shown in Fig. 3A, a correlation band of $1650 \mathrm{~cm}^{-1}$ appears at around 200, 400, $600 \mathrm{MPa}$, respectively, while the intensity of correlation at $200 \mathrm{MPa}$ is weaker than those at 400 and $600 \mathrm{MPa}$. A correlation band of $1626 \mathrm{~cm}^{-1}$ appears at around $600 \mathrm{MPa}$, but the intensity change is weaker than at $1650 \mathrm{~cm}^{-1}$. This indicates that the stability of $\beta$-sheet is stronger than that of the $\alpha$-helix in lysozyme upon pressure.

The weak correlation band at $1636 \mathrm{~cm}^{-1}$ appears at around $200 \mathrm{MPa}$ for HSA, and two other correlation bands related to 1626 and $1673 \mathrm{~cm}^{-1}$ appear at around $400 \mathrm{MPa}$, while that of $1656 \mathrm{~cm}^{-1}$ appears near $450 \mathrm{MPa}$. This indicates that the stability of the solvent exposed $\alpha$-helix $\left(1636 \mathrm{~cm}^{-1}\right)$ is lower than that of the solvent inaccessible $\alpha$-helix $\left(1656 \mathrm{~cm}^{-1}\right)$, which is composed of short turn connecting structures. The correlation band of $1626 \mathrm{~cm}^{-1}$ has a broad region from 200 to $600 \mathrm{MPa}$ in Fig. 3C. The strong band at $1639 \mathrm{~cm}^{-1}$ appeared at the center of $600 \mathrm{MPa}$, while a much weaker correlation band at $1673 \mathrm{~cm}^{-1}$ appeared at $400 \mathrm{MPa}$. It is sure that weak structural changes occurred at around $200 \mathrm{MPa}$. Figure 3D shows that the strong and broad correlation band positioned at $1639 \mathrm{~cm}^{-1}$ appears at around $200 \mathrm{MPa}$, and the weaker band at $1626 \mathrm{~cm}^{-1}$ comes into

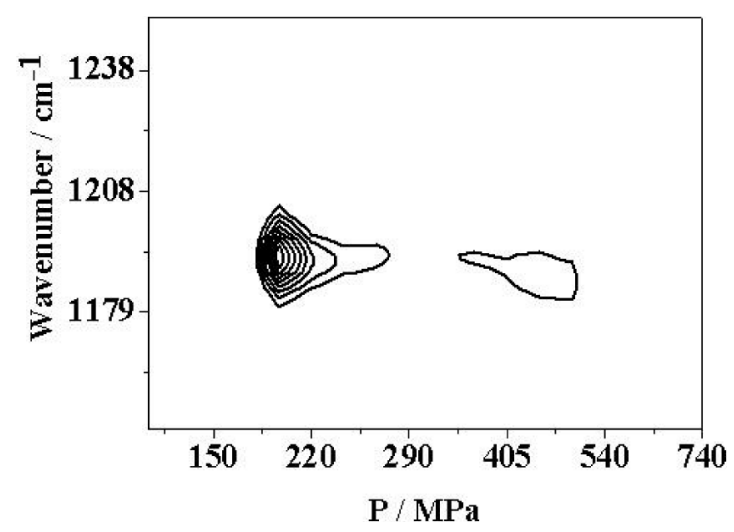

Fig. 4 MW2D correlation spectra of deuterium oxide under pressure.

existence at around $500 \mathrm{MPa}$. The two correlation bands at 1639 and $1626 \mathrm{~cm}^{-1}$ can mainly be attributed to $\beta$-sheets. Notably, the pressure-induced structural transitions of all four proteins are polymorphism processes.

Water and structural changes of four proteins under pressure

Pressure-induced changes of $\mathrm{D}_{2} \mathrm{O}$, a band center at $1208 \mathrm{~cm}^{-1}$ can be clearly observed under atmospheric pressure; this center is assigned to OD bending, ${ }^{21}$ and gradual shifts to lower wavenumbers with a pressure increase (Fig. S4, Supporting Information). MW2D calculation was preformed and is recorded in Fig. 4. It must be noted that two correlation bands centered at $1194 \mathrm{~cm}^{-1}$ can be observed at 200 and $400 \mathrm{MPa}$, respectively; the one at $200 \mathrm{MPa}$ is stronger than that at $400 \mathrm{MPa}$.

Zheng et al. ${ }^{22,23}$ investigated the absorbed band area change of water under pressure: they found that the structure change of water discontinues at 200 and $400 \mathrm{MPa}$, respectively. From the energy-dispersive X-ray diffraction technique, Okhulkov et al. ${ }^{24}$ found that the average separation between the nearest molecules decreases with pressure up to $\sim 200 \mathrm{MPa}$, but at higher pressures it begins to grow at $400-500 \mathrm{MPa}$. Raman scattering of the stretching band from water changes under pressure up to $600 \mathrm{MPa} ;{ }^{25}$ the result showed that the intensity decreased with increasing pressure initially and then reached a minimum at about $200 \mathrm{MPa}$, and increased again with the further increase about $400 \mathrm{MPa}$. These findings confirmed perfectly that local structure changes of water occurred at 200 and $400 \mathrm{MPa}$, in agreement with the current result of our MW2D analysis.

The above changes of water are highly consistent with the behaviors of four proteins under pressure. The water in the hydration shell of proteins has been proposed to play a crucial role in high pressure induced protein unfolding, and it was proposed that the penetration of water molecules into the hydrophobic core may induce unfolding of the system. ${ }^{26}$ The water penetration model was also supported by high pressure molecular dynamics simulations. ${ }^{27}$ Winter et al. ${ }^{28}$ carried out a series of molecular dynamics computer simulations on SNase. Their results indicated that the hydration of the interfacial hydrophobic part and the water density of the protein increased under pressure. Notably, slight changes of the H-bond network are observed at $200 \mathrm{MPa}$, probably due to the rearrangement of the residues at the protein surface. In addition, the structure change of water in the hydration shell of proteins was also well documented by results from scattering experiments. ${ }^{29}$ Those authors showed a wide range of non-random hydrogen bonding environments in the hydration shell of globular proteins. In addition to surface hydration, internal water molecules contribute 
also to protein stability. ${ }^{30}$ They provide the necessary flexibility for biological activity, act as a lubricant and render to the protein a certain level of plasticity. The presence of water molecules close to protein internal hydrogen bonds induces a lengthening of these hydrogen bonds. ${ }^{31}$ Therefore, it can be deduced that a structure change of water is an incentive that leads to changes of proteins when the pressure is up around $200 \mathrm{MPa}$.

In fact, similar phenomena also occur for other proteins as reported earlier. For example, high pressure NMR measurements of lysozyme indicated purely elastic deformation of the molecule in the $0.1-200 \mathrm{MPa}^{4}$ Marabotti et al. ${ }^{32}$ investigated pressure affects on the structure and the dynamics of D-galactose/ D-glucose-binding protein: a slope can be observed at $200 \mathrm{MPa}$ for the plot of wavenumber versus pressure, which was also observed in deconvoluted spectra of GGBP. Pfeiffer et al. ${ }^{33}$ reported an FTIR study on the pressure induced unfolding of lysozyme in pure $\mathrm{D}_{2} \mathrm{O}$, being close to the conditions of non-excess hydration. A similar phenomenon was also observed but it was not pointed out.

\section{Conclusions}

Pressure-induced structure changes of protein have been investigated by FTIR spectroscopy. The combination of the analysis of curve fitting, PCA and especially MW2D revealed information on structure transitions induced by pressure.

With regard to the pressure induced structure changes of lysozyme, HSA, ubiquitin, and RNase A, it became obvious that the characterized amide I band of protein shifted to lower wavenumbers with a common transition at $200 \mathrm{MPa}$, indicating that pressure induced a hydration increase at the lower pressure region. This is similar to the pressure induced structure change of pure water, and it is possible that the structure change in the proteins studied is caused by the structure change of water. Moreover, the change processes for the proteins were found not to be two-state processes, as several fluctuations along the pressure change were observed according to the analytical results of the PCA and MW2D analyses.

\section{Acknowledgements}

The authors are grateful for the financial supports of the Natural Science Foundation of China (Nos. 20934002 and 20973073), the National Basic Research Program (2007CB808006), Jilin Province Natural Science Foundation (20070926-01), the Programs for New Century Excellent Talents in University (NCET) and the 111 Project (B06009).

\section{Supporting Information}

The second derivative spectra, curve fitting, plots of position of amide I band versus pressure, and the pressure-induced IR spectra of deuterium oxide are available. This material is available free of charge on the Web at http://www.jsac.or.jp/ analsci/.

\section{References}

1. K. Heremans and L. Smeller, Biochim. Biophys. Acta, 1998, $1386,353$.

2. H. Imamura and M. Kato, Proteins: Struct., Funct., Bioinf.,
2009, 75, 911.

3. S. Chodankar, V. K. Aswal, J. Kohlbrecher, R. Vavrin, and A. G. Wagh, Phys. Rev. E, 2008, 77, 031901.

4. Y. O. Kamatari, H. Yamada, K. Akasaka, J. A. Jones, C. M. Dobson, and L. J. Smith, Eur. J. Biochem., 2001, 268, 1782.

5. K. Suzuki, Y. Miyosawa, and C. Suzuki, Arch. Biochem. Biophys., 1963, 101, 225.

6. D. W. Goddette, C. Paech, S. S. Yang, J. R. Mielenz, C. Bystroff, M. E. Wilke, and R. J. Fletterick, J. Mol. Biol., 1992, 228, 580.

7. M. A. Walsh, T. R. Schneider, L. C. Sieker, Z. Dauter, V. S. Lamzin, and K. S. Wilson, Acta Crystallogr., Sect. D: Biol. Crystallogr., 1998, 54, 522.

8. S. Sugio, A. Kashima, S. Mochizuki, M. Noda, and K. Kobayashi, Protein Eng., Des. Sel., 1999, 12, 439.

9. S. Vijiay-Kumar, C. E. Bugg, and W. J. Cook, J. Mol. Biol., 1987, 194, 531.

10. A. Wlodawer, N. Borkakoti, D. S. Moss, and B. Howlin, Acta Crystallogr., Sect. B: Struct. Sci., 1986, 42, 379.

11. Y. Wu, K. Murayama, and Y. Ozaki, J. Phys. Chem. B, 2001, 105, 6251.

12. Y. Wu, F. Meersman, and Y. Ozaki, Macromolecules, 2006 , 39, 1182.

13. P. T. T. Wong and D. J. Moffatt, Appl. Spectrosc., 1989, 43, 1279.

14. B. Czarnik-Matusewicz, K. Murayama, Y. Wu, and Y. Ozaki, J. Phys. Chem. B, 2000, 104, 7803.

15. K. Murayama, Y. Wu, B. Czarnik-Matusewicz, R. Tsenkova, and Y. Ozaki, J. Phys. Chem. B, 2001, 105, 4763.

16. M. Unger, S. Morita, H. Sato, Y. Ozaki, and H. W. Siesler, Appl. Spectrosc., 2009, 63, 1027.

17. P. I. Harris and D. Chapman, Biopolymers, 1995, 37, 251.

18. F. Meersman, L. Smeller, and K. Heremans, Biophys. J., 2002, 82, 2635.

19. K. R. Beebe, R. J. Pell, and M. B. Seasholtz, "Chemometrics: A Practical Guide", 1998, Wiley-Interscience, New York.

20. S. Morita, H. Shinzawa, R. Tsenkova, I. Noda, and Y, Ozaki, J. Mol. Struct., 2006, 799, 111.

21. S. Y. Venyaminov and F. G. Prendergast, Anal. Biochem., 1997, 248, 234.

22. H. F. Zheng, A. Shen, M. Zhang, Q. Sun, and J. Y. Chen, Prog. Nat. Sci., 2002, 12, 662.

23. L. R. Murphy, N. Matubayasi, V. A. Payne, and R. M. Levy, Fold. Des., 1998, 3, 105.

24. A. V. Okhulkov, Y. N. Demianets, and Y. E. Gorbaty, J. Chem. Phys., 1994, 100, 1578.

25. Q. Sun, H. Zheng, J. Xu, H. Xie, and E. Hines, Chin. Phys. Lett., 2003, 20, 445 .

26. A. Paliwal, D. Asthagiri, D. P. Bossev, and M. E. Paulaitis, Biophys. J., 2004, 87, 3479.

27. G. Hummer, S. Garde, A. E. Garcia, M. E. Paulaitis, and L. R. Pratt, Proc. Natl. Acad. Sci. U. S. A., 1998, 95, 1552.

28. N. Smolin and R. Winter, Biochim. Biophys. Acta, 2006, $1764,522$.

29. V. Makarov, B. M. Pettitt, and M. Feig, Acc. Chem. Res., 2002, 35, 376 .

30. K. Takano, J. Funahashi, Y. Yamagata, S. Fujii, and K. Yutani, J. Mol. Biol., 1997, 274, 132.

31. S. Fischer and C. S. Verma, Proc. Natl. Acad. Sci. U. S. A., 1999, 96, 9613.

32. A. Marabotti, A. Ausili, M. Staiano, A. Scirè, F. Tanfani, A. Parracino, A. Varriale, M. Rossi, and S. D'Auria, Biochemistry, 2006, 45, 11885.

33. H. Pfeiffer, K. Heremans, and M. Wevers, Chem. Phys. Lett., 2009, 469, 195. 